\title{
Wall Modeled Immersed Boundary Lattice Boltzmann Method for the Fluid-Structure Interaction of Ram-Air Parachutes and Paragliders
}

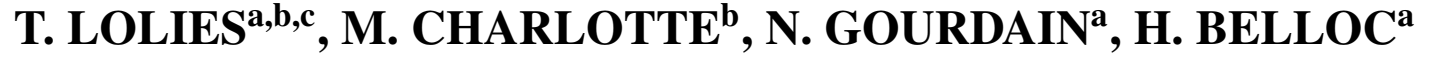 \\ a. Dpt. of Aerodynamics, Energetics and Propulsion, ISAE-Supaero, University of Toulouse, France, \\ b. University of Toulouse, ISAE, Institut Clement Ader - UMR CNRS 5312 \\ c. Bruce Goldsmith Design, research and development offices \\ Email : tom.lolies@isae-supaero.fr
}

\begin{abstract}
Ram-Air wings, or parafoils, are now used in various applications such as autonomous aerial delivery systems, kiteboats or paragliders. Developing reliable simulation tools to design these wings is a challenging task. Indeed, the structural integrity is not ensured by stiff mechanical parts within the canopy, but by the aerodynamic forces acting on itself. Fathoming such problems requires the use of fluid-structure interactions (FSI), where the deformation of the solid membrane forces the surrounding fluid to move accordingly, which in turn adjusts the pressure and friction forces on the solid in an iterative scheme. The immersed boundary method (IBM), a well-suited technique for FSI with large deformations, is used with a cartesian grid. Moreover, a highly efficient FSI framework is obtained coupling the IBM with the lattice-Boltzmann method (LBM), as presented in [1]. The LBM proved to be one of the most efficient and promising strategy to perform large eddy simulations of weakly compressible flows [2]. A major challenge when using a cartesian grid without dynamic remeshing procedures is that the entire geometry and its displacements need to be encapsulated in a large volume of constant mesh size. Therefore resolving the strong gradients pertaining to the turbulent boundary layer would involve unacceptable simulation costs. To deal with this issue, an IBM specific wall model based on an integral boundary layer is developed. Using a conventional serial staggered procedure, the fluid solver is coupled to a mass-spring damper system, a suitable cost-efficient alternative to finite element methods for largely deformable non linear thin membranes [3]. An aerodynamic validation test case, a NACA0012 wing at Re $6.10^{5}$, is presented. Then, simulation results of a complete parachute in steady state flight are compared to wind tunnel experiments, to validate the deformed shape of the canopy and the aerodynamic coefficients.
\end{abstract}

\section{References}

[1] K. Suzuki, K. Minami, and T. Inamuro, "Lift and thrust generation by a butterfly-like flapping wingbody model: Immersed boundary-lattice Boltzmann simulations," Journal of Fluid Mechanics, vol. 767, pp. 659-695, 2015.

[2] R. Löhner, "Towards overcoming the LES crisis," International Journal of Computational Fluid Dynamics, vol. 33, pp. 87-97, mar 2019.

[3] M. Chen and F. J. Boyle, "Investigation of membrane mechanics using spring networks: Application to red-blood-cell modelling," Materials Science and Engineering C, vol. 43, pp. 506-516, oct 2014. 